\title{
DEVELOPMENT OF A HIGH-SPEED VIDEOGRAMMETRIC MEASUREMENT SYSTEM WITH APPLICATION IN LARGE-SCALE SHAKING TABLE TEST
}

\author{
S. $\mathrm{Gao}^{1}$, Z. $\mathrm{Ye}^{1, *}, \mathrm{C} . \mathrm{Wei}^{1}, \mathrm{X} . \mathrm{Liu}^{2}, \mathrm{X} . \mathrm{Tong}^{1}$ \\ ${ }^{1}$ College of Surveying and Geo-Informatics, Tongji University, Shanghai 200092, China \\ - (1410900,89_yezhen, cwei, xhtong)@tongji.edu.cn \\ ${ }^{2}$ Key Laboratory for Urban Geomatics of National Administration of Surveying, Mapping and Geoinformation, \\ Beijing University of Civil Engineering and Architecture, Beijing 100048, China - liuxianglei@ bucea.edu.cn
}

\section{ICWG II/III: Pattern Analysis in Remote Sensing}

KEY WORDS: High-speed camera, Videogrammetric system, Image sequence processing, Shaking table test

\begin{abstract}
:
The high-speed videogrammetric measurement system, which provides a convenient way to capture three-dimensional (3D) dynamic response of moving objects, has been widely used in various applications due to its remarkable advantages including noncontact, flexibility and high precision. This paper presents a distributed high-speed videogrammetric measurement system suitable for monitoring of large-scale structures. The overall framework consists of hardware and software two parts, namely observation network construction and data processing. The core component of the observation network is high-speed cameras to provide multiview image sequences. The data processing part automatically obtains the $3 \mathrm{D}$ structural deformations of the key points from the captured image sequences. A distributed parallel processing framework is adopted to speed up the image sequence processing. An empirical experiment was conducted to measure the dynamics of a double-tube five-layer building structure on the shaking table using the presented videogrammetric measurement system. Compared with the high-accuracy total station measurement, the presented system can achieve a sub-millimeter level of coordinates discrepancy. The 3D deformation results demonstrate the potential of the non-contact high-speed videogrammetric measurement system in dynamic monitoring of large-scale shake table tests.
\end{abstract}

\section{INTRODUCTION}

In the field of civil engineering, the engineering structure should be evaluated by vibration test, wind tunnel, collapse and other tests to assess the structural quality and safety factor. Automatic and precise measurement of the dynamic deformations of some key points on the structure is crucial to these tests for further analysis. In general, the contact sensors such as displacement gauges, strain gauges, and accelerometers are commonly used for deformation measurement. However, these conventional sensors have limitations such as limited measurement range, ease of damage or failure, increased model quality, and time-consuming and laborious installation (Ribeiro et al., 2014, Ye et al., 2018).

Videogrammetry, as an alternative to the conventional sensors, is an extended non-contact $3 \mathrm{D}$ vision metrology technique that expands the methods and models of photogrammetry from still imagery to multiple time steps (Lin et al., 2008). The videogrammetric system and the vision-based system uses highspeed and high-resolution cameras to measure the spatial information changes of high-speed moving objects in a noncontact manner and accurately reflect the deformation characteristics, overcoming many defects of traditional contact sensors. It is able to realize 3D multipoint and wide-range measurement as well as is capable of repetitive measurements and permanent records. Therefore, it is drawing increasing attention in both static and dynamic measurement (Fraser, Riedel, 2000, Maas, Hampel, 2006, Liu et al., 2015). In (Chang, Ji, 2007), the authors used two commercial-grade digital video cameras to measure 3D structural vibration response for three

\footnotetext{
${ }^{*}$ Corresponding author
}

experimental tests in the laboratory. In (Tang et al., 2010), a 3D digital image correlation system was developed for smallscale deformation measurement in experimental mechanics. In (Leifer et al., 2011), three synchronized CCD cameras was adopted to measure the acceleration of high-speed moving objects mounted on a modal shaker, which verified the feasibility of videogrammetric technique in the shaking table test. (Tong et al., 2017) proposed a high-speed videogrammetric system and designed a specific artificial target to monitor a progressive collapse test of a single-layer lattice shell. In (Ye et al., 2018), the authors presented a practical vision-based system to measure the 3D structural vibration deformations of shaking table tests with a landslide dam model.

However, the observation objects of most of previous experiments are small structures or local areas of large structures. The videogrammetric measurement with small field of view cannot directly adapt to the overall deformation analysis in largescale civil engineering tests. In order to realize the effective and robust deformation monitoring in large-scale and widerange tests, all of observation network, data processing and system implementation should be specifically considered. Therefore, this paper presents a practical high-speed videogrammetric measurement system to measure the multipoint 3D deformations of larger structures. The system consists of a hardware system with multiple high-speed and high-resolution synchronized cameras, an automatic data processing workflow using advanced photogrammetry and computer vision algorithms, and a distributed parallel processing framework to accelerate the calculation. An empirical experiment of monitoring of largescale shaking table tests with a double-tube five-floor building structure is carried out to validate the performance and reliabil- 
ity of the presented videogrammetric system.

\section{METHODOLOGY}

In Fig. 1, the overall framework of the presented videogrammetric measurement system is illustrated, which includes hardware and software two parts: observation network construction and data processing. Here, data processing part involving retrieving the 3D deformations of the target points from image sequences with a distributed parallel processing framework is the main contribution of our system.

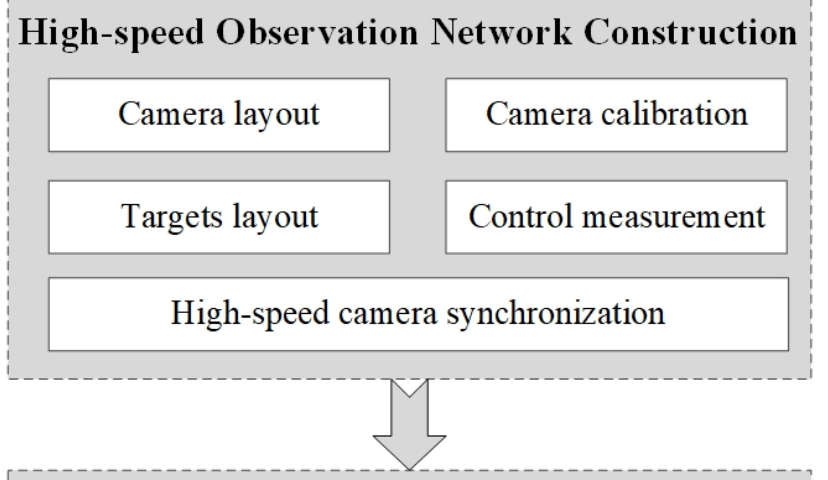

Videogrammetric Data Processing

Image enhancement of image sequence

\begin{tabular}{|c|}
\hline Target recognition and matching on the first frame \\
\hline Target tracking for each image sequence \\
\hline Bundle adjustment and 3D coordinates calculation \\
\hline \\
\hline \\
\hline Deformation parameters estimation \\
\hline
\end{tabular}

Figure 1. The overall framework of the presented videogrammetric measurement system.

\subsection{Observation network}

The observation network is the hardware components of the videogrammetric systems. Several high-performance hardware devices are required, such as high-speed cameras, synchronous controllers, data acquisition cards, and data storage cards. The high-speed camera (see 2 for a sample) plays a most important role, whose capabilities directly decide the potential of videogrammetric system. In general, the selection of the appropriate cameras is driven by requirements in accuracy, resolution, acquisition frame rate, synchronization, amount of data, spectral information, field of view, scale, and cost (Luhmann, 2010). In this study, certain industrial cameras with high stability, high frame rate, high transmission capacity and high anti- interference ability are selected. The cameras should be carefully positioned and orientated to ensure the field of view fully cover the measured objects. Prior to the measurement, the cameras are independently calibrated to obtain the interior orientation parameters and the lens distortion parameters. The cameras are fixed during the tests, and then the camera parameters keep constant at each epoch for facilitating the following photogrammetric analysis. In order to acquire and store largecapacity image data in real time, each high-speed camera needs to be equipped with a high-performance image acquisition card and a high-speed storage disk array. A synchronous controller is also necessary to keep the simultaneous image acquisition of all the connected high-speed cameras taken at the same time.

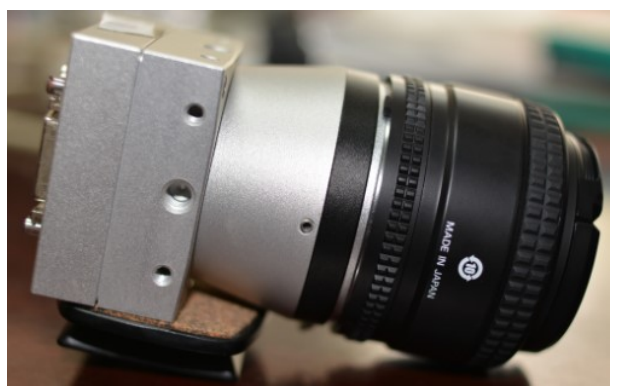

Figure 2. A sample of high-speed camera.

In addition, the control information is indispensable for photogrammetric analysis to provide the absolute references of scale and orientation. A total station is typically used to measure the $3 \mathrm{D}$ spatial coordinates of control points and establish the control network constructed by these control points attached to some stationary objects. Artificial targets are commonly applied in videogrammetric measurement in order to efficiently track the key positions of the structural models. These targets can be automatically detected for all images, and are significantly useful in the case that natural targets are hard to attain by feature detection. A circular target was chosen as tracking point and control point in this study because of its robustness in identification and tracking. As shown in Fig. 3, the tracking target is composed of a white circle and a black background and is pasted on the structure surface to represent the key positions. The control target adds a cross-wire, a inner-circle design and retro-reflective material on the centre to improve the precision of total station measurement. The size of the artificial targets should be determined by the model size, field of view, camera resolution and so on. In general, the radius of the circle in the artificial target is set as about 20 pixels on the captured images.

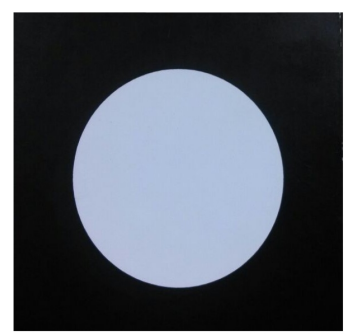

(a)

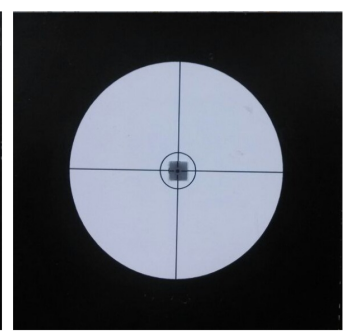

(b)
Figure 3. A sample of artificial target: (a) tracking target and (b) control target. 


\subsection{Data processing}

2.2.1 Image sequence processing The objective of image sequence processing is to automatically obtain the image coordinates of the targets at each epoch. The workflow of our image sequence processing is shown in Fig. 4, which includes image enhancement, target recognition, target matching and target tracking. The image enhancement and target tracking is performed on all the captured image sequences, while the target recognition and matching is only need to be performed on the first frame of each image sequence.

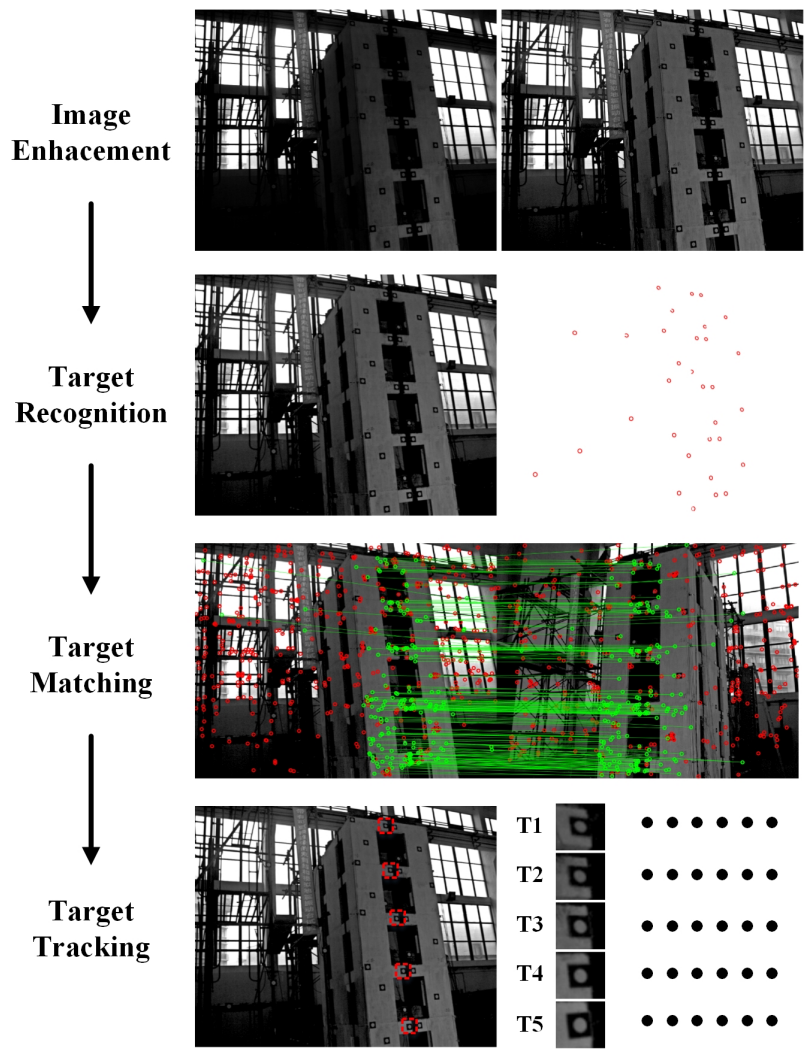

Figure 4. The workflow of image sequence processing.

As the artificial light source is unsuitable in the case of monitoring large structures, image enhancement is alternatively adopted on every frame to ensure the good illumination and contrast conditions (Hong et al., 2019). A local color correction algorithm (Gomila Salas, Lisani, 2011) for contrast enhancement is used to reduce the dark areas and enrich the texture details.

For the used circular artificial targets, target recognition on the initial frame is automatically achieved by ellipse detection. In this study, a state-of-the-art elliptical arc detector denoted as ELSDc (Ptrucean et al., 2017) is applied. The elliptical primitives are determined through candidate generation by region growing and chaining, model validation and selection by a contrario theory. The ellipse outputs are then filtered by restricting the axes and delimiting angles. The finally extracted ellipse centers represent the key positions to be measured.

The targets separately detected on the first frame of each image sequences should be matched in order to retrieve the $3 \mathrm{D}$ coordinates. This target matching is hard to realize only using the targets information due to the viewpoint difference and depth variation (Hong et al., 2015). Therefore, we resort to featurebased registration that detects and matches additional salient features to obtain a projective transformation model between images. We use Harris-Laplace detector (Mikolajczyk, Schmid, 2004), SIFT descriptor (Lowe, 2004) and Multi-GS algorithm (Chin et al., 2012) for feature detection, feature descriptor and outlier removal, respectively. With this transformation model, the target point can find the corresponding target points on other images as the nearest points relative to the warped positions.

In addition, the detected target position is also used to provide a initial reference for the following target tracking. Correlationbased template matching can be adopted to estimate the 2D motion between a local template centered at the target position in the first frame and a deformed copy in successive frames (Ye et al., 2019). In this study, least squares matching method (Gruen, 1985) is selected for accurate target tracking. This method considers the geometric deformation of template and can achieve the tracking results with high accuracy and high reliability. After image sequence processing, the 2D image coordinates of the corresponding targets on all frames of each captured image sequence can be obtained.

2.2.2 3D reconstruction In the process of $3 D$ reconstruction, the estimation of the exterior orientation parameters including the position and orientation of the cameras is the most important part, which affects the accuracy of the 3D coordinates calculation. Using the target positions on the first frames and the know 3D spatial coordinates of control points, we can obtain the the exterior orientation parameters using bundle adjustment. Its basic model is a collinearity equation (see Eq.(1)), which establishes the perspective relationship between the $2 \mathrm{D}$ projection and the 3D space. Based on the results of camera calibration, target recognition and matching, and total station measurement, the final solutions of bundle adjustment are achieved by an iterative least squares estimation process. As the cameras remain stable during the measurement process, the interior and exterior orientation parameters are constant for all epochs. Therefore, based on the target tracking results and the interior and exterior orientation parameters, the 3D spatial coordinates of the tracking points can be easily calculated through forward intersection, which is also based on the collinearity equation.

$$
\left\{\begin{array}{l}
x-x_{0}+\Delta x=-f \frac{a_{1}\left(X_{A}-X_{S}\right)+b_{1}\left(Y_{A}-Y_{S}\right)+c_{1}\left(Z_{A}-Z_{S}\right)}{a_{3}\left(X_{A}-X_{S}\right)+b_{3}\left(Y_{A}-Y_{S}\right)+c_{3}\left(Z_{A}-Z_{S}\right)} \\
y-y_{0}+\Delta y=-f \frac{a_{2}\left(X_{A}-X_{S}\right)+b_{2}\left(Y_{A}-Y_{S}\right)+c_{2}\left(Z_{A}-Z_{S}\right)}{a_{3}\left(X_{A}-X_{S}\right)+b_{3}\left(Y_{A}-Y_{S}\right)+c_{3}\left(Z_{A}-Z_{S}\right)}
\end{array}\right.
$$

where $x, y$ are the image coordinates of the target; $x_{0}, y_{0}, f$ are the interior orientation parameters; $\Delta x, \Delta y$ are the lens distortions; $X_{S}, Y_{S}, Z_{S}$ are the spatial coordinates of the perspective center of the camera; $X_{A}, Y_{A}, Z_{A}$ are the spatial coordinates of the target; $a_{i}, b_{i}, c_{i}(i=1,2,3)$ are the elements of the rotation matrix of the camera.

2.2.3 Deformation parameter estimation Deformation parameters such as displacement, velocity and acceleration are important parameters to describe the motion state of moving objects. Therefore, after acquiring the 3D spatial coordinates of each target, the deformation parameters at the key positions can be further solved by the numerical differentiation with the corresponding 3D spatial coordinates. High-frequency noise is inevitable caused by inherent measurement uncertainty such as camera synchronization and image processing. A smoothing filter (such as a Savitzky-Golay filter) is adopted to remove the high-frequency noise while preserving the true features of the process prior to the deformation estimation (Leifer et al., 2011). 
The displacement values of a target are the difference between the 3D spatial coordinates at epoch $n$ and at the first epoch, and are calculated as:

$$
\left\{\begin{array}{c}
S_{X_{n}}=X_{n}-X_{0} \\
S_{Y_{n}}=Y_{n}-Y_{0} \\
S_{Z_{n}}=Z_{n}-Z_{0}
\end{array}\right.
$$

where $S_{X_{n}}, S_{Y_{n}}$, and $S_{Z_{n}}$ denote the displacement values at epoch $n$ in the $X, Y$, and $Z$ directions, respectively; $X_{0}, Y_{0}$, $Z_{0}$ and $X_{n}, Y_{n}, Z_{n}$ represent the $3 \mathrm{D}$ coordinates in the $X, Y$, and $Z$ directions at the first epoch and at epoch $n$, respectively.

The 3D velocity values of a target at epoch $n V_{X_{n}}, V_{Y_{n}}$, and $V_{Z_{n}}$ are calculated as:

$$
\left\{\begin{aligned}
V_{X_{n}} & =\frac{X_{n+1}-X_{n-1}}{2 \Delta T} \\
V_{Y_{n}} & =\frac{Y_{n+1}-Y_{n-1}}{2 \Delta T} \\
V_{Z_{n}} & =\frac{Z_{n+1}-Z_{n-1}}{2 \Delta T}
\end{aligned}\right.
$$

where $X_{n+1}, Y_{n+1}$, and $Z_{n+1}$ are the 3D coordinates in the three directions at epoch $n+1 ; X_{n-1}, Y_{n-1}$, and $Z_{n-1}$ are the $3 \mathrm{D}$ coordinates in the three directions at epoch $n-1 ; \Delta T$ indicates the time interval between adjacent epochs.

Similarly, the 3D acceleration values of a target at epoch $n a_{X_{n}}$, $a_{Y_{n}}$, and $a_{Z_{n}}$ are calculated as:

$$
\left\{\begin{aligned}
a_{X_{n}} & =\frac{V_{X_{n+1}}-V_{X_{n-1}}}{2 \Delta T} \\
a_{Y_{n}} & =\frac{V_{Y_{n}+1}-V_{Y_{n-1}}}{2 \Delta T_{Z_{n-1}}} \\
a_{Z_{n}} & =\frac{V_{Z_{n+1}}}{2 \Delta T}
\end{aligned}\right.
$$

where $V_{X_{n+1}}, V_{Y_{n+1}}$, and $V_{Z_{n+1}}$ are the velocity values in the three directions at epoch $n+1 ; V_{X_{n-1}}, V_{Y_{n-1}}$, and $V_{Z_{n-1}}$ are the velocity values in the three directions at epoch $n-1$.

\subsection{Distributed parallel computing}

In order to achieve the purpose of on-site computing, the parallel computing is necessary to improve the computing efficiency (Gao et al., 2019). In our measurement system, each high-speed camera is equipped with a minicomputer, and a principal controller is used to manage these minicomputers. In the minicomputer, the high-performance multi-core Central Processing Unit (CPU) is applied to support the parallel computing of the target matching and tracking of massive image sequences, which will take a lot of time in data processing. The targets will be partitioned into different groups according to the core numbers of CPUs so that every group can be processed and calculated in parallel. The distributed computing parallel framework is shown in Fig. 5. The processing steps are depicted as follows. (1) The initial frames are sent to principle controller from minicomputer. (2) The initial corresponding target points are calculated through target recognition and feature-based matching. (3) These corresponding points are transferred to the corresponding minicomputer respectively. (4) The tracking and matching of target points can be computed in parallel. (5) The sequential image coordinates of corresponding points will be sent to principle controller to implement 3D reconstruction.

\section{RESULTS AND DISCUSSION}

\subsection{Experimental details}

In this study, experiments that monitor the dynamic response of a double-tube five-layer reinforced concrete core tube struc-

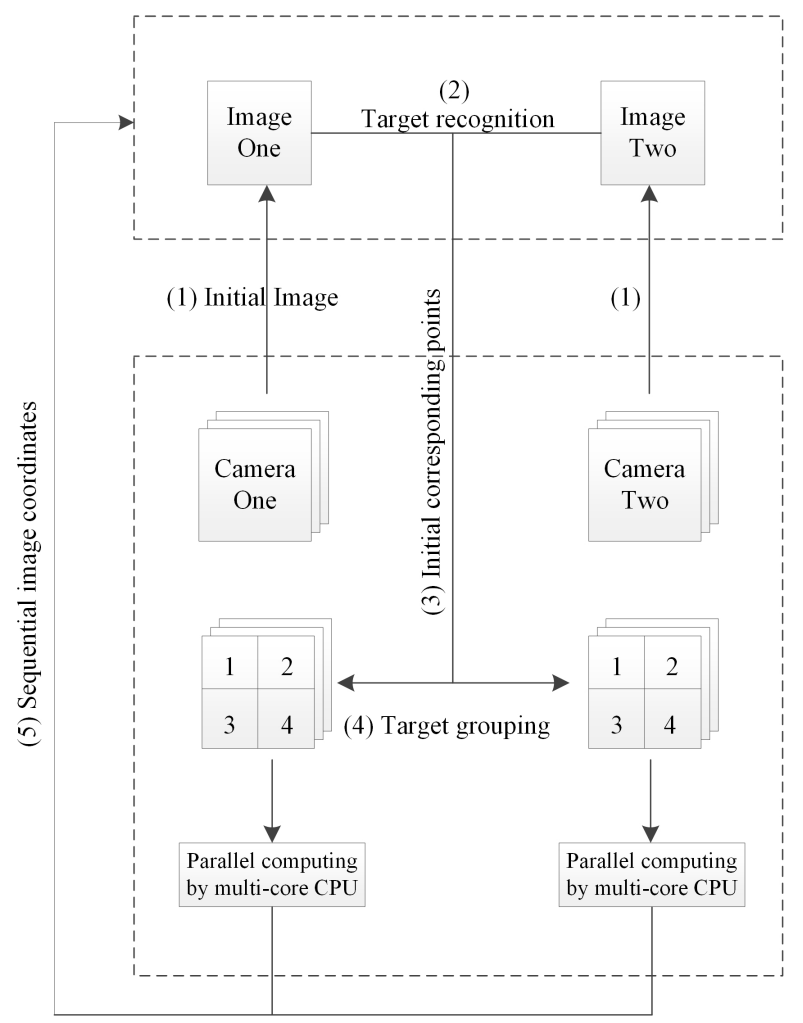

Figure 5. Distributed computing parallel framework.

ture on a large-scale shaking table (see Fig. 6) were conducted to demonstrate the feasibility of the videogrammetric measurement system. The size of the structural model is $2420 \mathrm{~mm} \times$ $1620 \mathrm{~mm} \times 4500 \mathrm{~mm}$ with a model scale ratio of $1 / 5$. A largescale $3 \mathrm{D}$ shaking table with a platform size of $4 \mathrm{~m} \times 4 \mathrm{~m}$ was employed for the tests.

A set of six high-speed cameras were used in the observation network to capture around the entire frame, and each two high-speed cameras formed a pair of stereoscopic observations. The cameras used are the CL600 $\times 2$ CMOS camera produced by Optronis $\mathrm{GmbH}$, Germany. The image size is $1280 \times 1024$ pixels, and the frame rate was set as $200 \mathrm{fps}$. Each camera was equipped with a $20 \mathrm{~mm}$ fixed focus lens to increase the field of view, and was mounted on a tripod to keep it stable. During the shaking table test, six high-speed cameras simultaneously captured and stored image sequences. Circular artificial targets with a diameter of $50 \mathrm{~mm}$ were pasted on the model and objects to represent tracking and control points. The key position of each layer has one tracking point (see the red rectangles in Fig. 6(b)). In addition, a control network was arranged with some control points positioned on the surrounding stationary scaffolds, and the 3D spatial coordinates of these control points were measured by a Sokkia NET05AX total station with a 0.5 $\mathrm{mm}$ distance measurement accuracy to provide a reference system and to assess the performance of videogrammetric measurement.

\subsection{Comparison with total station measurement}

In order to evaluate the spatial coordinate accuracy obtained from the videogrammetric measurement, the control points are divided into two sets, one set is inputted into bundle adjustment to provide the control information, and another one is selected as check points whose $3 \mathrm{D}$ coordinates are calculated using 
videogrammetric data processing as well. The absolute discrepancy between the videogrammetric measurement and the total station were estimated through these check points, which is shown in Table 1 . The root mean square values of absolute discrepancy are $0.29 \mathrm{~mm}, 0.18 \mathrm{~mm}$ and $0.20 \mathrm{~mm}$ in the $X, Y$, and $Z$ direction, respectively. The accuracy satisfies the requirement of the experiment.

\subsection{Results of deformation parameter estimation}

In this experiment, a seismic wave in the $Y$ direction was inputted into the shaking table. Therefore, we mainly displayed the vibration parameters in the $Y$ direction. The displacement results of the tracking points on different layers were estimated using the developed data processing workflow, which are shown in Fig. 7(a). These displacement time histories describe the 3D detailed morphological responses of the structure during the vibration process. In addition, the velocity and acceleration time histories of the tracking points can also be obtained, as shown in Fig. 7(b) and Fig. 7(c). It can be seen from the results that the spatial motion trends of the tracking points in all layers are almost similar. The deformation results of the structure obtained by the presented videogrammetric measurement system provide an important basis for the subsequent structural mechanics analysis.

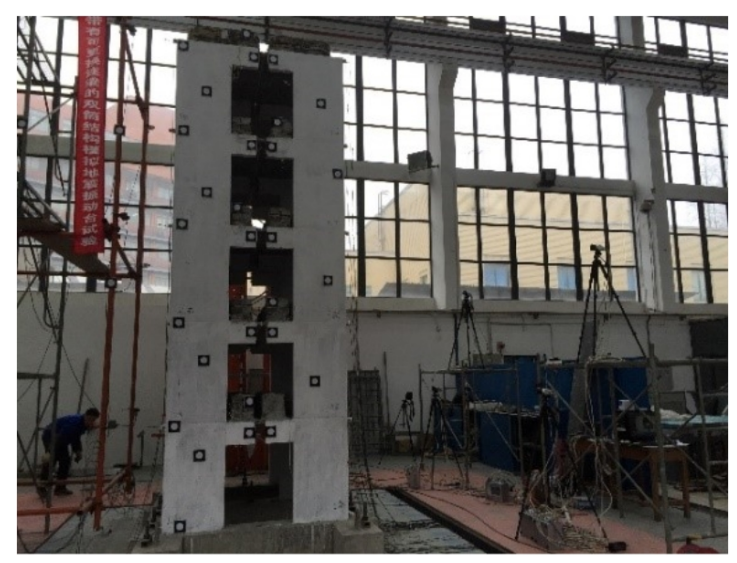

(a)

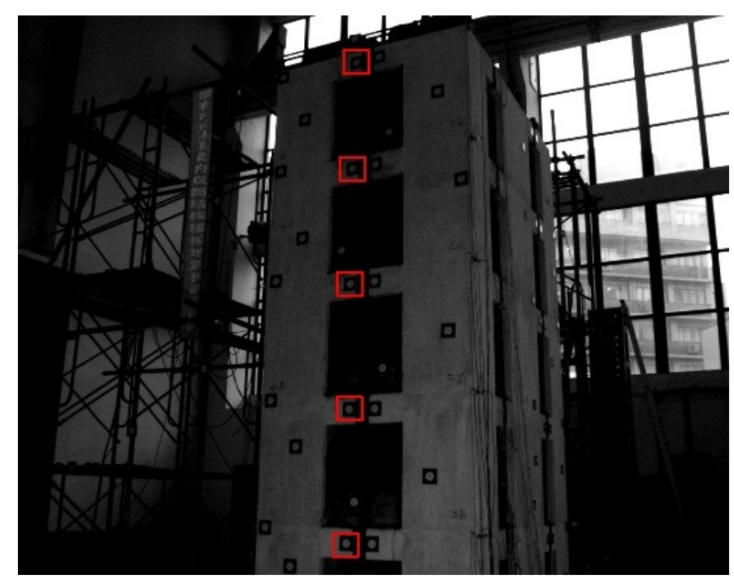

(b)

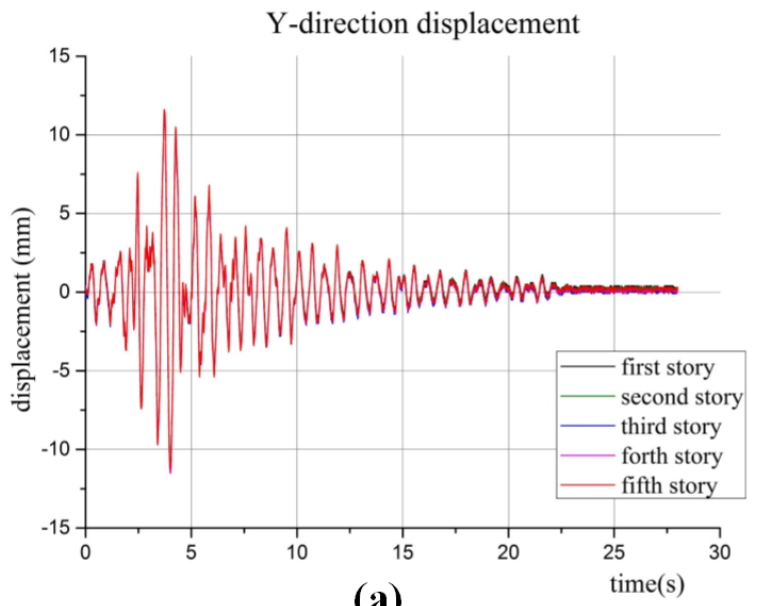

(a)

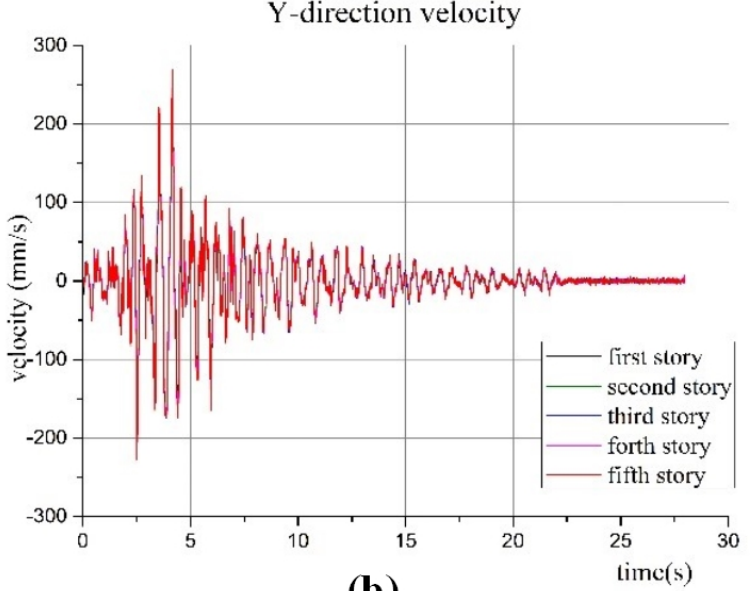

(b)

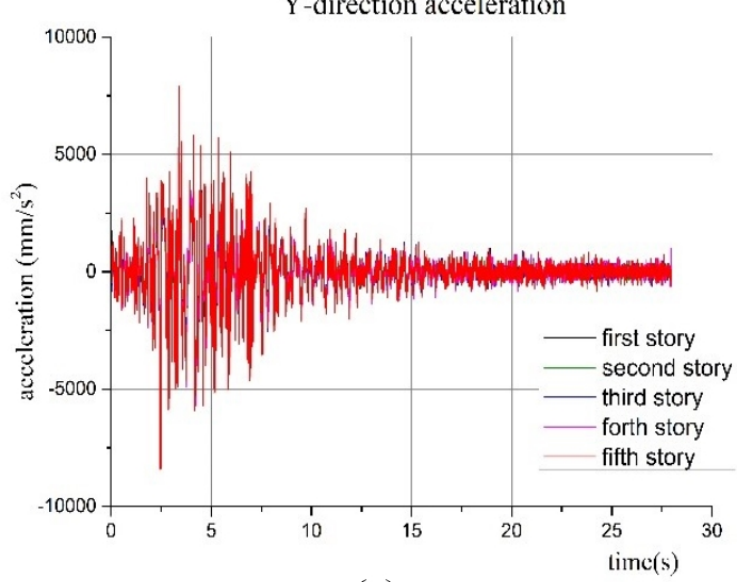

(c)

Figure 7. Results of deformation parameter estimation in the $Y$ direction. (a) Displacement time histories, (b) velocity time histories, and (c) acceleration time histories of five tracking points.

Figure 6. Scene of experiments. (a) Testing site and (b) sample image from the high-speed camera. 


\begin{tabular}{|l|c|c|c|}
\hline \multirow{2}{*}{ No. } & \multicolumn{3}{|c|}{ Discrepancy values } \\
\cline { 2 - 4 } & $\Delta X(\mathrm{~mm})$ & $\Delta Y(\mathrm{~mm})$ & $\Delta Z(\mathrm{~mm})$ \\
\hline 1 & 0.19 & 0.13 & 0.11 \\
2 & -0.38 & -0.19 & -0.23 \\
3 & -0.26 & -0.16 & 0.25 \\
4 & 0.36 & -0.18 & 0.17 \\
5 & -0.30 & 0.12 & 0.28 \\
6 & -0.21 & 0.24 & 0.09 \\
7 & -0.35 & -0.23 & -0.11 \\
8 & 0.22 & -0.16 & -0.25 \\
RMS & 0.29 & 0.18 & 0.20 \\
\hline
\end{tabular}

Table 1. Coordinate difference values of the check points

\section{CONCLUSION}

Non-contact videogrammetric technique is gradually adopted in different fields due to its advantages. This paper focuses on the application of high-speed videogrammetric technique in the dynamic monitoring of large-scale structures. A practical highspeed videogrammetric measurement system is presented, and the hardware and software components of the system including a observation network, an automatic data processing workflow and a distributed parallel processing framework are introduced. An actual large-scale shaking table test with a doubletube five-layer structure was conducted to validate the power of high-speed videogrammetric measurement system for monitoring the $3 \mathrm{D}$ vibration response. In the experiment, six synchronized high-speed cameras at a frame rate of $200 \mathrm{fps}$ were employed. Compared with the high-accuracy total station measurements, the videogrammetric system was able to achieve a sub-millimetre accuracy in the 3D spatial coordinates calculation. The displacement, velocity and acceleration of tracking points were successfully obtained by the videogrammetric measurement, which detailed reflect $3 \mathrm{D}$ dynamic responses of structural vibration. The experimental results show that the non-contact videogrammetric system is an alternative to monitor the large-scale structure.

\section{REFERENCES}

Chang, C., Ji, Y., 2007. Flexible videogrammetric technique for three-dimensional structural vibration measurement. Journal of engineering mechanics, 133(6), 656-664.

Chin, T., Yu, J., Suter, D., 2012. Accelerated hypothesis generation for multistructure data via preference analysis. IEEE Transactions on Pattern Analysis and Machine Intelligence, 34(4), 625-638.

Fraser, C.S., Riedel, B., 2000. Monitoring the thermal deformation of steel beams via vision metrology. ISPRS Journal of Photogrammetry and Remote Sensing, 55(4), 268-276.

Gao, S., Tong, X., Chen, P., Ye, Z., Hu, O., Wang, B., Zhao, C., Liu, S., Xie, H., Jin, Y., Xu, X., Liu, S., Wei, C., 2019. Full-field deformation measurement by videogrammetry using self-adaptive window matching. The Photogrammetric Record, 34(165), 36-62.

Gomila Salas, J.G., Lisani, J.L., 2011. Local color correction. Image Processing On Line, 1, 260-280.

Gruen, A., 1985. Adaptive least squares correlation: a powerful image matching technique. South African Journal of Photogrammetry, Remote Sensing and Cartography, 14(3), 175-187.
Hong, D., Liu, W., Su, J., Pan, Z., Wang, G., 2015. A novel hierarchical approach for multispectral palmprint recognition. Neurocomputing, 151, 511 - 521.

Hong, D., Yokoya, N., Chanussot, J., Zhu, X.X., 2019. An augmented linear mixing model to address spectral variability for hyperspectral unmixing. IEEE Transactions on Image Processing, 28(4), 1923-1938.

Leifer, J., Weems, B., Kienle, S.C., Sims, A.M., 2011. Threedimensional acceleration measurement using videogrammetry tracking data. Experimental Mechanics, 51(2), 199-217.

Lin, S.-Y., Mills, J.P., Gosling, P.D., 2008. Videogrammetric monitoring of as-built membrane roof structures. The Photogrammetric Record, 23(122), 128-147.

Liu, X., Tong, X., Yin, X., Gu, X., Ye, Z., 2015. Videogrammetric technique for three-dimensional structural progressive collapse measurement. Measurement, 63, 87-99.

Lowe, D.G., 2004. Distinctive image features from scaleinvariant keypoints. International Journal of Computer Vision, 60(2), 91-110.

Luhmann, T., 2010. Close range photogrammetry for industrial applications. ISPRS Journal of Photogrammetry and Remote Sensing, 65(6), 558 - 569.

Maas, H.-G., Hampel, U., 2006. Photogrammetric techniques in civil engineering material testing and structure monitoring. Photogrammetric Engineering \& Remote Sensing, 72(1), 3945.

Mikolajczyk, K., Schmid, C., 2004. Scale \& affine invariant interest point detectors. International Journal of Computer Vision, 60(1), 63-86.

Ptrucean, V., Gurdjos, P., Grompone von Gioi, R., 2017. Joint a contrario ellipse and line detection. IEEE Transactions on Pattern Analysis and Machine Intelligence, 39(4), 788-802.

Ribeiro, D., Calada, R., Ferreira, J., Martins, T., 2014. Noncontact measurement of the dynamic displacement of railway bridges using an advanced video-based system. Engineering Structures, 75, 164 - 180.

Tang, Z., Liang, J., Xiao, Z., Guo, C., Hu, H., 2010. Threedimensional digital image correlation system for deformation measurement in experimental mechanics. Optical Engineering, 49(10), 1 - 9 - 9 .

Tong, X., Gao, S., Liu, S., Ye, Z., Chen, P., Yan, S., Zhao, X., Du, L., Liu, X., Luan, K., 2017. Monitoring a progressive collapse test of a spherical lattice shell using high-speed videogrammetry. The Photogrammetric Record, 32(159), 230-254.

Ye, Z., Tong, X., Xu, Y., Gao, S., Liu, S., Xie, H., Chen, P., Lu, W., Liu, X., 2018. An improved subpixel phase correlation method with application in videogrammetric monitoring of shaking table tests. Photogrammetric Engineering \& Remote Sensing, 84(9), 579-592.

Ye, Z., Tong, X., Zheng, S., Guo, C., Gao, S., Liu, S., Xu, X., Jin, Y., Xie, H., Liu, S., Chen, P., 2019. Illuminationrobust subpixel Fourier-based image correlation methods based on phase congruency. IEEE Transactions on Geoscience and Remote Sensing, 57(4), 1995-2008. 